\title{
Effect of Mechanical Stress on Characteristics of Silicon Thermal Oxides
}

\author{
Jui-Yuan Yen, Chia-Hong HUANG and Jenn-Gwo HwU*
}

Rm 446, Department of Electrical Engineering/Graduate Institute of Electronics Engineering, National Taiwan University,

Taipei, Taiwan, Republic of China

(Received July 23, 2001; accepted for publication September 13, 2001)

The characteristics of oxides grown on silicon wafers under external mechanical stress were studied. It was found that the $\mathrm{SiO}_{2}$ films grown under external compressive stress had a decreased etching rate. From capacitance-voltage $(C-V)$ measurements, it was observed that a tensile sample had a reduced interface state density, while that of a compressive sample was increased. [DOI: 10.1143/JJAP.41.81]

KEYWORDS: oxides, mechanical stress, interface state density

As silicon technology continues to be promoted and the feature size of devices is continuously scaled down to the deep sub-quarter-micrometer regime, the trend of scaling and integration has brought attention to the fundamental and physical limits of devices. Therefore, the issue of reliability of mechanical stress becomes critical since the devices have become much more complex in terms of geometry and process steps. Many defects and failures of integrated circuits can be traced ultimately to mechanical stress that occurs at various process steps. ${ }^{1)}$ Mechanical stress generally occurs due to the deposition of different kinds of films such as $\mathrm{SiO}_{2}$, poly-Si, $\mathrm{Si}_{3} \mathrm{~N}_{4}$ and metal electrodes. The structure of devices at sharp corners and interconnect edges exhibits high stress as well. Mechanical stress causes not only the dislocation of the silicon substrate, cracking or failure of thin films, but also the degradation of electrical characteristics. It has been proposed that compressive and tensile stresses from temperature nonuniformity or mechanically applied forces may affect oxidation kinetics and rates, ${ }^{2-4)}$ and there is still interest in studying the mechanical stress effect on the electrical characteristics of devices further. In this work, we report the stress effects based on etching rate and interface trap density data.

P-type, (100)-oriented, 3-inch silicon wafers with a resistivity of $1-10 \Omega \cdot \mathrm{cm}$ were used in this experiment. After standard cleaning followed by $\mathrm{HF}\left(\mathrm{H}_{2} \mathrm{O}: \mathrm{HF}=50: 1\right)$ dip and DI water rinse, the wafers were oxidized many times in a furnace with a dilute oxygen flow $\left(\mathrm{O}_{2}: \mathrm{N}_{2}=1: 1\right)$ at $900-950^{\circ} \mathrm{C}$, at $1 \mathrm{~atm}$. The wafers were placed vertically on a quartz boat. A small slice of quartz about $20 \mathrm{~g}$ was placed across the middle of two adjacent wafers so that the two adjacent wafers sustained compressive and tensile stress, respectively, as shown in the inset of Fig. 1. After oxidation, the oxide thickness was measured using an automaticscanning ellipsometer with an accuracy of $\pm 5 \AA$. No postoxidation annealing was carried out on the grown oxides. MOS capacitors $150 \mu \mathrm{m} \times 150 \mu \mathrm{m}$ were fabricated by photolithography and wet etching. Evaporated aluminum films were used as gate electrodes and also as back ohmic contacts after back oxide removal. No post-metallization annealing was carried out on the samples for electrical measurements in this work.

The $\mathrm{SiO}_{2}$ films of our samples were repeatedly dipped in $1 \%$ dilute $\mathrm{HF}$ solution and the thickness of the remaining oxides was measured using an automatic-scanning ellipsometer at $5 \mathrm{~mm}$ pitch. Figure 1 shows the etching results of

\footnotetext{
*Corresponding author. E-mail address: hwu@cc.ee.ntu.edu.tw
}

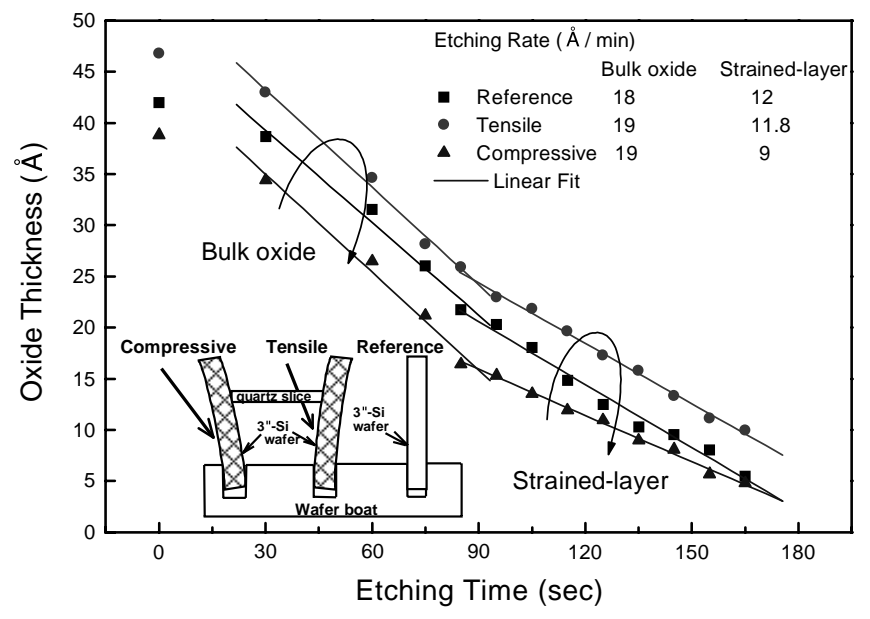

Fig. 1. Etching results and average etching rates of bulk and strainedlayer oxides for compressive, tensile, and reference samples. The inset showed the experimental setup.

oxide thickness versus etching time for the above three samples. It is noted that each value of oxide thickness in the figures is taken as the average thickness of measured points by the ellipsometer over a central $2 \mathrm{~cm}^{2}$ area of silicon wafers. From the figures, one can see the decreased etching rates of three samples when the oxide thickness is less than approximately $20 \AA$. The etching rates of bulk oxides and the strained layer are also calculated, as shown in this figure. From Fig. 1, we can see that all three samples have a strained-layer oxide near the $\mathrm{Si}-\mathrm{SiO}_{2}$ interface. ${ }^{5,6)}$ The etching rates of the bulk oxides of the three samples are nearly equal to $18-19 \AA / \mathrm{min}$. However, the etching rate of strained layers for the compressive sample is different from the others, i.e., the etching rate of the compressive sample is slightly lower than that of the reference or tensile sample. This can possibly be explained by the following. Because there is an approximate $125 \%$ volume expansion from $\mathrm{Si}$ to $\mathrm{SiO}_{2}$ during thermal oxidation, ${ }^{7-13)}$ all the $\mathrm{SiO}_{2}$ films grown on silicon substrates are observed in a state of compressive stress, except when the oxidation temperature is as high as about $1100^{\circ} \mathrm{C} .^{14,15)}$ When the silicon substrate is oxidized under tensile stress, the $\mathrm{SiO}_{2}$ film grows downward freely, and is observed to have a high oxidation rate. The $\mathrm{SiO}_{2}$ film on a tensile Si substrate is likely to have the same strainedlayer structure as that of the reference sample since it is grown without constraint, as shown by our etching results. However, when $\mathrm{SiO}_{2}$ film is grown on a silicon substrate 
under compressive stress, the mismatch of lattice constants between $\mathrm{SiO}_{2}$ and $\mathrm{Si}$ will be enhanced. Therefore, it is possible to form more $\mathrm{Si}$ dangling bonds at the $\mathrm{Si} / \mathrm{SiO}_{2}$ interface to create a Si-rich suboxide layer. Thus, the etch rate of such oxides should be decreased.

The interface state density is the most important factor determining the quality of $\mathrm{SiO}_{2}$ films. High-quality $\mathrm{SiO}_{2}$ films must have low interface state density for use as gate dielectric material. Midgap interface trap density $\left(D_{\text {itm }}\right)$ and flat-band voltage $\left(V_{\mathrm{fb}}\right)$ were extracted from the high- and low-frequency capacitance-voltage $(C-V)$ curves. A mechanism based on experimental results was proposed by $\mathrm{Ma}$ and Hook ${ }^{16,17)}$ in which interface trap generation could be explained by the broken bond model. The broken bond model suggests that a strained $\mathrm{Si}-\mathrm{O}$ or $\mathrm{Si}-\mathrm{Si}$ bond broken by capturing an incident carrier tends to propagate toward the $\mathrm{Si}-\mathrm{SiO}_{2}$ interface due to the gradient of bond strain, which is found to be present within an interfacial region of about $3 \mathrm{~nm}$. This will finally result in interface-trap generation. Since larger strains exists at the $\mathrm{Si}-\mathrm{SiO}_{2}$ interface for thicker oxides, more traps are generated at the $\mathrm{Si}-\mathrm{SiO}_{2}$ interface. $\mathrm{Ma}^{16)}$ observed that $D_{\text {itm }}$ increases with oxide thickness. Our control samples with different thickness are also in agreement with the experiments and model proposed by Ma. ${ }^{16)}$ Figure 2 compares the $D_{\text {itm }}$ for oxides grown under different stress conditions. The inset of Fig. 2 shows the low-frequency $C-V$ curves for gate oxides grown on silicon substrates with compressive, tensile, and no stress. The $C-V$ curves are measured on devices within a central $1.5 \mathrm{~cm}^{2}$ area of silicon wafers. It is naturally expected that $D_{\text {itm }}$ increases with oxide thickness. However, in our work, the $80 \AA$ oxides of the compressive sample have higher $D_{\text {itm }}$ than the $87 \AA$ oxides of the reference sample (with no applied stress),

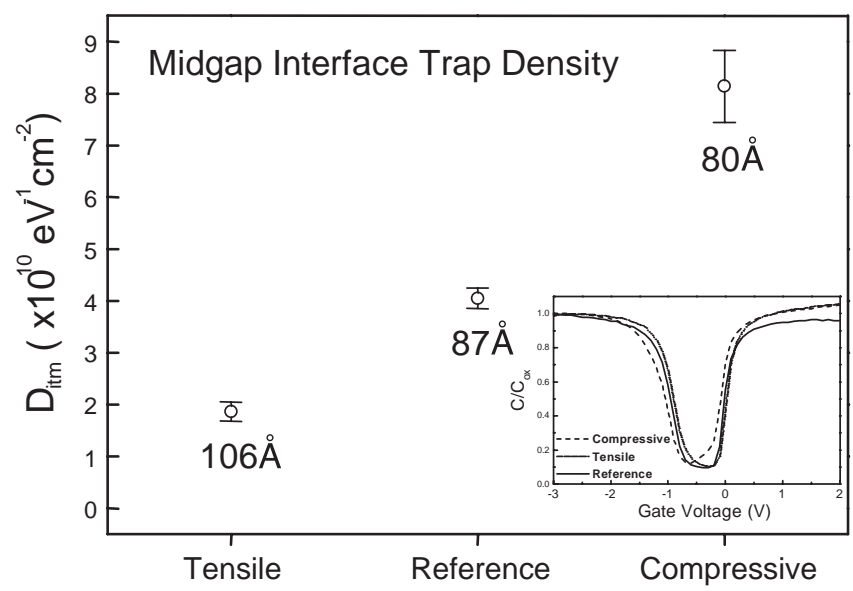

Fig. 2. Comparison of $D_{\text {itm }}$ for oxides grown under applied compressive, tensile, and no stress. The inset shows the low-frequency capacitance $(C$ $V)$ curves for oxides grown on silicon substrates under different stresses. while the $106 \AA$ oxides of the tensile sample have lower $D_{\text {itm }}$ than those of the reference sample. Clearly, the results of Ma and those of our control samples are different. This can be explained by the fact that the $D_{\text {itm }}$ is affected by the stress applied on the $\mathrm{Si}$ substrates. It is believed that our experimental results are in complete contrast to the wellaccepted broken bond model; the difference in $D_{\text {itm }}$ is mainly caused by the applied mechanical stress. Based on the physical model of the $\mathrm{Si}-\mathrm{SiO}_{2}$ interface and the discussions in our previous studies, it is suggested that a tensile stress on the silicon surface will enlarge the atom spacing of silicon and the oxygen atoms become more easily diffused and connect more easily to the dangling bonds. However, a compressive stress will shorten the atom spacing of silicon and leave a greater numbers of dangling bonds at the $\mathrm{Si}-$ $\mathrm{SiO}_{2}$ interface during oxidation. Therefore, it is reasonable to consider that the $D_{\text {itm }}$ of a tensile sample is lower than that of a compressive one.

Silicon oxidation with externally applied mechanical stress is useful in studying stress-related problems. In this work, the effects of externally applied mechanical stresses on the characteristics of silicon thermal oxides have been investigated. This is important in the investigation of oxide quality under stress especially for next generation silicon technology.

This work was supported by the National Science Council of the Republic of China under Contract No. NSC 89-2215E-002-085.

1) S. M. Hu: J. Appl. Phys. 70 (1991) R53.

2) A. Minhalyi, R. J. Jaccodine and T. J. Delph: Appl. Phys. Lett. 74 (1999) 1981

3) C. K. Huang, R. J. Jaccodine and S. R. Butler: Silicon Nitride and Silicon Dioxide Thin Insulating Film, eds V. J. Kapoor and K. T. Hankins (Electrochemical Society, Pennington, NJ, 1987) Vol. 87-10, p. 343.

4) R. Deaton and H. Z. Massoud: J. Appl. Phys. 70 (1991) 3588

5) K. Eriguchi, Y. Harada, and M. Niwa: IEEE Int. Electron Devices Meet., 1998, p. 175.

6) K. Eriguchi, Y. Harada and M. Niwa: J. Appl. Phys. 87 (2000) 1990

7) D. B. Kao, J. P. McVittie, W. D. Nix and K. C. Saraswat: IEEE Trans. Electron Devices 34 (1987) 1008.

8) D. B. Kao, J. P. McVittie, W. D. Nix and K. C. Saraswat: IEEE Trans. Electron Devices 35 (1988) 25.

9) T. J. Delph: J. Appl. Phys. 83 (1998) 786.

10) E. A. Irene, E. Tierney and J. Angiello: J. Electrochem. Soc. 129 (1982) 2594

11) L. M. Mack, A. Reisman and P. K. Bhattacharya: J. Electrochem. Soc. 136 (1989) 3433.

12) T. Y. Tan and U. Gosele: Appl. Phys. Lett. 39 (1981) 86.

13) B. J. Mrstik, A. G. Revesz, M. Ancona and H. L. Hughes: J. Electrochem. Soc. 134 (1987) 2020.

14) E. P. EerNisse: Appl. Phys. Lett. 35 (1979) 8.

15) E. P. EerNisse: Appl. Phys. Lett. 30 (1977) 290.

16) T. P. Ma: Appl. Phys. Lett. 27 (1975) 615.

17) T. B. Hook and T. P. Ma: Appl. Phys. Lett. 48 (1986) 1208. 